\title{
Can stimulus enhancement explain the apparent success of the model-rival technique in the domestic dog (Canis familiaris)?
}

\author{
$1 \quad$ Nina R. Cracknell, Daniel Simon Mills*, Patricia Kaulfuss \\ Animal Behaviour, Cognition and Welfare Group, Department of Biological Sciences, University of Lincoln, \\ Riseholme Park, Lincoln LN2 2LG, UK
}

Accepted 14 April 2008

\begin{abstract}
The model-rival technique is a method of training whereby an animal learns the distinguishing features of a target object, such as name and colour, by observing a trainer and a potential competitor engage in conversation about these features. In this study the apparent effectiveness of the model-rival technique in training dogs to perform a selection-retrieval task by McKinley and Young [McKinley, S., Young, R.J., 2003. The efficacy of the model-rival method when compared with operant conditioning for training domestic dogs to perform a retrieval-selection task. Appl. Anim. Behav. Sci. 81, 357-365] was investigated to evaluate the hypothesis that simpler forms of learning may be responsible for the results. This was tested by repeating McKinley and Young's model-rival training method and comparing the results to those of training sessions devised to include different forms of stimulus enhancement of the object to be retrieved. These training sessions involved: minimal enhancement, during which the experimenters made no interactions with the target object; indirect stimulus enhancement, during which both experimenters switched their gaze between the dog and the target object; or direct stimulus enhancement, during which one of the experimenters held the target object. It was found that only the model-rival and direct enhancement methods resulted in a significant number of dogs successfully completing the selection-retrieval test. There was also evidence to suggest that with the direct stimulus enhancement training method dogs learned quicker than with the model-rival training method. It was concluded that dogs are able to learn to retrieve a named object in a selection-retrieval task as a result of simple stimulus enhancement, without necessarily understanding the complex cognitive processes which underpin learning in the model-rival process.

(C) 2008 Published by Elsevier B.V.
\end{abstract}

Keywords: Dog; Learning; Model-rival; Stimulus enhancement; Training

\footnotetext{
* Corresponding author. Tel.: +44 1522 895356; fax: +44 1522895328.

E-mail address: dmills@lincoln.ac.uk (D.S. Mills).
}

0168-1591/\$ - see front matter (C) 2008 Published by Elsevier B.V.

doi:10.1016/j.applanim.2008.04.004

Please cite this article in press as: Cracknell, N.R. et al.,Can stimulus enhancement explain the apparent success of the model-rival technique in the domestic dog (Canis familiaris)?, Appl. Anim. Behav. Sci. (2008), doi:10.1016/j.applanim.2008.04.004 


\section{Introduction}

The interpretation of results from animal cognition research can be a problem as there may be a temptation to accredit results to complex cognitive abilities on the part of the animal, by enthusiastic proponents of animal cognition. That is not to say that animals do not possess such higher cognitive skills, but simply that, taking into account the methodologies used, the data gained does not warrant such conclusions and results might be better explained by simpler cognitive processes. This is the principle of Lloyd Morgan's canon, according to which we should not ascribe a behaviour to a more complex cognitive process if a simpler one is sufficient to account for what we observe (Pearce, 1999).

A classic example of a behaviour which has been ascribed to more complex mechanisms of learning than necessary is sweet potato washing in Japanese macaques. Originally, this was proposed to have spread through the troop by imitation (Kawai, 1965), a form of learning that is thought to require quite complex cognitive abilities, however after reanalysing the data Galef (1992) argues that it is more likely to have spread by a process of local or stimulus enhancement.

A recent study by McKinley and Young (2003) claimed effectiveness for the model-rival technique in dog training. The model-rival technique involves using social stimuli to create an interest in a target object without the use of food or other rewards and has been popularised by Irene Pepperberg through her training and testing of the cognitive ability of Alex, an African Grey parrot (Pepperberg, 1999). During these training sessions Alex was encouraged to answer questions about a target object, which were put to him by an experimenter. A second experimenter then either demonstrated the correct response or an incorrect response. For a correct response the experimenter was praised and received the target object to play with, and for an incorrect response the experimenter was punished. This second experimenter acted as a model for Alex by answering the first experimenter's questions, but also acted as a rival with Alex for the first experimenter's attention and the chance to play with the target object (Pepperberg, 1999). After observing the interactions of the two experimenters Alex was invited to join in with the questions. Alex only received the target object and the first experimenter's attention when he answered correctly. If Alex answered incorrectly the first experimenter directed their attention away from Alex and back to the second experimenter (Pepperberg, 1999).

As dogs do not have the same communicative abilities as parrots McKinley and Young (2003) used a variation on the model-rival technique set out by Pepperberg. In their training sessions the dog was sat in front of two experimenters who were talking animatedly about a target object which they passed between them. The name of the target object was spoken at the end of each sentence during the training session, for example "isn't this a lovely pair of SOCKS?" These training sessions were intended to teach the dog the name of the object by making the dog 'want' the object the experimenters were apparently interested in. At the end of the training session the target object was placed in a line-up with two other objects and the dog was asked to retrieve it using the object's name, for example "fetch the SOCKS" (McKinley and Young, 2003). McKinley and Young (2003) found that the results from their version of the model-rival technique were comparable to those obtained by traditional operant conditioning techniques which are commonly used in dog training. It was therefore concluded that the model-rival technique was an effective method of dog training.

However, a review of the methodologies used by McKinley and Young (2003) suggests that the dogs might have learned to pick the target object as a result of stimulus enhancement, without understanding of the social and verbal context which underpin the model-rival method. Stimulus enhancement is involved in some forms of social learning, and describes the phenomenon 
whereby an animal is more likely to interact with a stimulus that it has previously seen a group member interacting with (Wynne, 2001). It could therefore be argued that the dogs tended to pick the target object in McKinley and Young's (2003) study because they had seen the two experimenters interacting with it, not because they understand the interaction between the two experimenters at any level.

The results of the McKinley and Young (2003) investigation also show that dogs would discriminate object labels on the basis of shape. This discriminatory process was investigated further in our study by testing if dogs discriminated between objects of the same shape but of different colour (or luminance), having learned the identity of a particular object of a particular colour. This part of the study was carried out on dogs that passed the initial part of the experiment, and thus had shown that they could discriminate on the basis of object shape.

The aim of this study was firstly to investigate whether simple stimulus enhancement could explain the apparent effectiveness of the model-rival technique in dog training reported by McKinley and Young (2003) and compare performance using a variety of methods of enhancement. The second aim of the study was to investigate whether, having learned to retrieve a particular named object of a certain colour, the dogs discriminated between this and similar objects of a different colour.

\section{Methods}

\subsection{Animals}

A convenience sample of 10 pet dogs were recruited from an advertisement in the local press. The group consisted of 7 females and 3 males, with ages ranging from 3 to 8 years, with a mean of 5.4 years. Among the 10 were 3 pastoral dogs, 3 terrier dogs, 3 gundogs and 1 crossbreed. Before testing began all owners were briefed about the study and their written informed consent was obtained. All dogs were tested to make sure they could 'sit', 'stay' and 'fetch' on command.

\subsection{Toys}

The objects used in the retrieval-selection task consisted of three sets of three types of rubber toy. These were a bone, a dumbbell and a folded "tugger" (see Fig. 1). All toys were between 11.5 and $14 \mathrm{~cm} \mathrm{long.} \mathrm{The}$ toys were chosen so that they were of similar size and colour but were different shapes so the dogs could easily distinguish between them. One set was bright yellow, one dark blue, and the other medium red in order to be able to offer objects with distinctly different colours and luminance in different training sessions, whilst accounting for the fact that dogs are believed to be red-green colour blind (Neitz et al., 1989). Each test contained a set of toys of the same colour to control for any bias towards or against certain colours. All toys were washed, between the training sessions and before the retrieval task took place, in an enzymatic cleaning solution and then rinsed in clean water, to control for any odour cues from the 'target' object.

\subsection{Target object and target word}

The toy used in each training session was referred to as the 'target object'. The target object and target object colour were assigned randomly so each dog undertook each training session with different coloured and shaped objects to avoid biases that might have come from the physical appearance of the target object. Regardless of which toy was chosen the name given to the target object was also assigned randomly for each dog, for each training session, using four labels novel to the dogs: 'goom', 'faf', 'pipe', and 'jid'. These words were chosen as each word had three phonemes, with each phoneme being semantically distinct from the equivalent phoneme in the other commands, since it has been shown that dogs can detect changes to the 


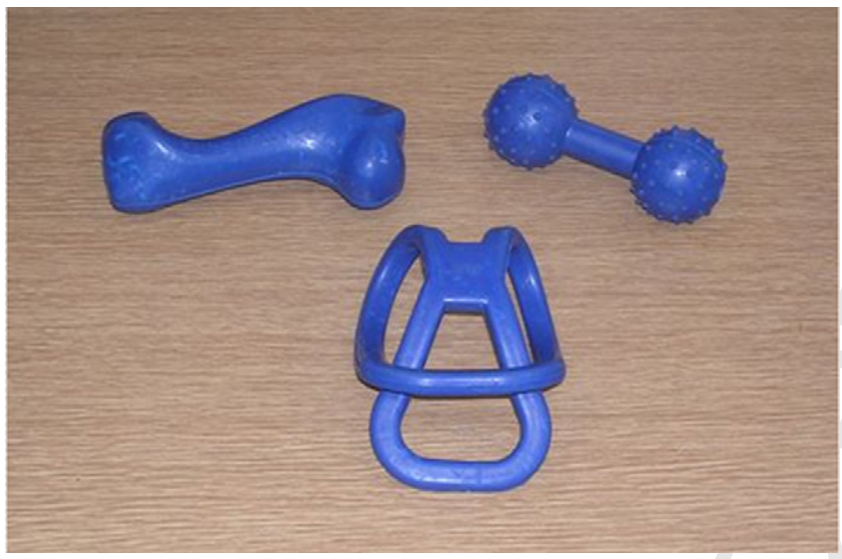

Fig. 1. Example of toys sets used.

phonemes of words (Fukuzawa et al., 2005). If different target words contained similar phonemes this might lead to interference from learning the name of one object on the subsequent learning of the name of another object. This control also meant that each dog was given equivalent length 'target words', eliminating bias that may occur through differences in pronunciation or word length. The experiment consistent of three parts: training sessions and selection retrieval tests, and discrimination tests.

\subsection{Training sessions}

Four different training sessions were used in this study, with a different object and label used in each session. The methods are described in detail below, with the order being described first. Five of the dogs were randomly assigned to McKinley and Young's model-rival training method to complete first, while the other 5 dogs undertook a minimal enhancement training method first. The procedures were then crossed so all dogs undertook both methods.

It was necessary to start with the model-rival and minimal enhancement training methods first as the model-rival method was the reference treatment and the minimal enhancement method was the simplest level of stimulus enhancement that might explain the results of the model-rival technique. If a significant proportion of dogs had learned successfully using minimal enhancement it would have been irrelevant to test the dogs at greater levels of stimulus enhancement, to test the hypothesis that they could learn through this process.

On the dogs' next visit, which was always at least a week after their first visit, 5 dogs were randomly assigned to an indirect stimulus enhancement training method to complete first, while the other 5 dogs were assigned to a direct stimulus enhancement training method. The training methods were again crossed so that all dogs undertook all four training methods.

Before each training session the dogs were allowed to acclimatise to the training room for 10 min with both the experimenter and the assistant present. This was to reduce the distraction of the dogs during the training sessions. Only the 'target object' was present during the training sessions and rubber gloves were worn when handling the toys to avoid the transfer of experimenter odour cues. The same two experimenters conducted all training sessions.

The basic set up of the training sessions was the same for each training method (see Fig. 2), and followed the methodologies used by McKinley and Young so that experiments could be compared. The dog was secured on a lead and sat $2 \mathrm{~m}$ away from the experimenter and the assistant who were sat in front of the dog. Each training session lasted for $2 \mathrm{~min}$. After being exposed to the training method the dog was asked to retrieve the target object when it was placed $2 \mathrm{~m}$ away from the dog, using a verbal command which 


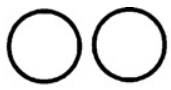

D

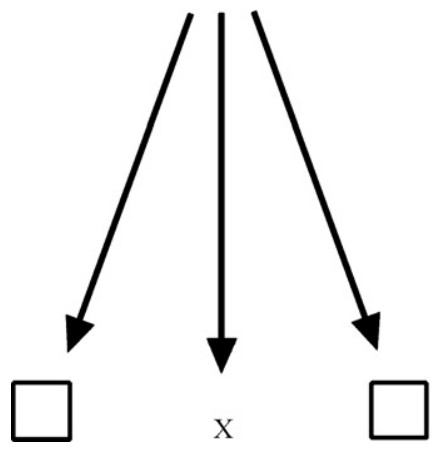

D Position of dog

$\bigcirc$ Position of experimenters during retrieval

Position of experimenters during training

X Position of toy(s) for retrieval

Distance of 2 metres

Fig. 2. Positioning of experimenters and dog during training sessions. D: position of dog; $\bigcirc$ : position of experimenters during retrieval; $\square$ : position of experimenters during training; X: position of toy(s) for retrieval; $\downarrow$ : distance of $2 \mathrm{~m}$.

included the name of the target object. This retrieval attempt took place immediately after the training session. The verbal command was given only once at which point the dog had $30 \mathrm{~s}$ to retrieve the object. If the dog failed to retrieve the target object the training period was repeated and the extra time was added to the total training time. The procedure was repeated as necessary a maximum of 15 times $(30 \mathrm{~min}$ of exposure). If the dog could not retrieve the object after this time, it was recorded as a learning failure. If the dog successfully retrieved the object it was given verbal praise. The dog was not allowed to touch the target object during any of the training methods but simply observed any interactions taking place between the experimenter, the assistant and the target object.

\subsection{Model-rival training (McKinley and Young, 2003)}

An assistant acted as the model-rival. The experimenter and the assistant discussed the target object, the name of which always appeared last in the sentence. This consisted of a standard dialogue between the experimenter and the model-rival. For example:

- Experimenter: Can you see the GOOM? Hands object to the model-rival.

- Model-rival: Yes, thank you for the GOOM-Hands object back to experimenter, etc.

This dialogue continued for the 2 min of the training session, during which time the target object was handed back and forth between the experimenter and the assistant. As a standard dialogue was used we were sure that the target word was spoken the same number of times for each dog.

In order to keep the dog interested the experimenter and model-rival spoke in a highly animated way. They both looked at the target object during training, but ensured that voice and body orientation were directed towards the dog.

\subsection{Minimal enhancement training}

A screen was placed between the dog and the experimenter and the target object was put into position so that the dog did not see the experimenter interacting with it. The target object was placed on the floor in between the experimenter and the assistant. The screen was then pulled back and the experimenter took her 
place. Both the experimenter and the assistant sat still and looked at the dog. At no point during the training did either the experimenter or the assistant look at or make any gestures towards the target object, as it has been shown that dogs attend to human gaze (Hare and Tomasello, 1999). The body orientation of the experimenter and the assistant was directed towards the dog throughout the session. Thus the first time the dog heard the object label was during the retrieval task.

\subsection{Indirect stimulus enhancement}

A screen was placed between the dog and the experimenter and the target object was put into position so that the dog did not see the experimenter interacting with it. The target object was placed on the floor between the experimenter and the assistant. The screen was then pulled back and the experimenter took her place. During training both the experimenter and the assistant switched their eye contact from the dog to the target object, thus indirectly, through their gaze, potentially enhancing the target object stimulus. When eye contact was switched between the dog and the target object the head was moved to face the direction of the gaze. Apart from this exchange of eye contact no other gestures were made towards the target object. The body orientation of the experimenter and the assistant was directed towards the dog during the session.

\subsection{Direct stimulus enhancement}

The experimenter held the target object in her hands. The experimenter kept eye contact on the target object and the assistant sat still, keeping eye gaze towards the dog. At no point during the training did either the experimenter or the assistant make any other gestures towards the target object. The body orientation of the experimenter and assistant was directed towards the dog during the session.

\subsection{Selection-retrieval tests}

In all cases the object of the test was to see if the dog would consistently retrieve the 'target object' from a group of three similar sized and similar coloured objects, having demonstrated its ability to retrieve it in the initial training sessions. This was a test of the dogs' prospective memory. Prospective memory is memory for actions to be performed in the future. This is used when there is an interruption between the intention and the action (Schacter, 2001).

After the particular training session had been successfully completed, the dog was removed from the training room briefly by the experimenter while the 'target object' was placed in a line-up with two other toys of the same colour. The objects were positioned $1 \mathrm{~m}$ away from each other in a line, with the initial position of the target object determined from a random sequence. The dog was then brought back into the room to begin the retrieval test.

To ensure that each dog started the test the same distance away from the object line-up, and that the toys in the line-up were always the same distance away from each other, the positions were marked on the floor, with tape. The toys were positioned $1 \mathrm{~m}$ apart and set in an arc shape so that each toy was the same distance from the dog (see Fig. 3). Each dog began the selection-retrieval test at a distance of $2 \mathrm{~m}$ away from the toy line-up, with both the experimenter and the assistant behind the dog so as not to distract it or influence its choice in the test. The dog was released at the beginning of the test by the experimenter using the verbal command 'fetch the GOOM', 'fetch the FAF', 'fetch the PIPE' or 'fetch the JID' depending on which target word had been assigned to the object previously. This command was only given once and the dog was allowed $30 \mathrm{~s}$ to retrieve the correct object.

The first test was complete when the dog had retrieved an object from the line-up and returned it to the experimenter. If the dog retrieved the wrong item, the object was taken from the dog without praise, if the correct item was retrieved, the object was taken and the dog was verbally praised. This process was repeated with the target object in a second position in the line-up and repeated again with it in a third position in the line-up. The dog was briefly removed from the training room by the assistant while the experimenter reset the line-up for both the second and third attempts. Thus, the dog had to retrieve the target object from all 


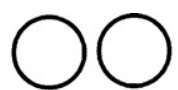

$\mathrm{D}$

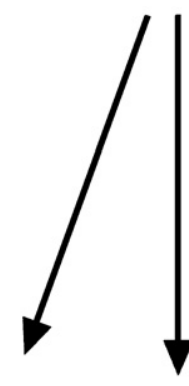

$\mathrm{X}$

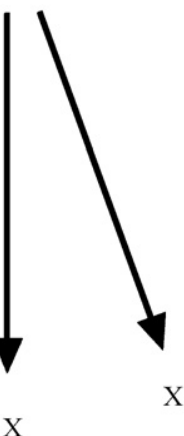

D Position of dog

Position of experimenters during retrieval

Position of experimenters during training

Position of toy(s) for retrieval

Distance of 2 metres

Fig. 3. Positioning of experimenters and dog during selection-retrieval test. D: position of dog; $\bigcirc$ : position of experimenters during retrieval; $\square$ : position of experimenters during training; X: position of toy(s) for retrieval; $\downarrow$ : distance of $2 \mathrm{~m}$.

three positions in the line-up. If, after all three tests, the dog had failed to retrieve the target object on one or more occasions the training session was repeated and the extra time was added to the total training time for that training method. A maximum training time of $30 \mathrm{~min}$ was allowed in total, including initial and repeated training sessions, thus a maximum of 15 training sessions were allowed to complete a maximum of 10 attempts at the selection-retrieval task. Toys were washed in enzymatic washing liquid and rinsed in clean water in between each attempt at the line-up to remove any odour cues and rubber gloves were worn whilst handling the toys.

\subsection{Discrimination tests}

8 of the original 10 dogs took part in the discrimination test. These were dogs that had passed at least one of the previous selection-retrieval tests. These dogs underwent another test to examine the specificity of their learning and discriminatory resolution of the target object, in a new situation.

The discrimination test took the form of one more attempt by the dog at the selection-retrieval task, except this time the target object was placed in a line-up with two other objects that were the same shape, but were different in colour. Dogs are believed to be red-green colour blind (Neitz et al., 1989), so the three colours of toy used were dark blue, bright yellow and medium red, meaning the dogs were able to discriminate using a rule based on either colour or luminance. The target object's position in the line-up was randomly assigned for each dog.

The selection-retrieval task then took place as before using the target word used in the selection-retrieval test which the dog had previously passed. The discrimination test took place after every training method that the dog passed using the target item and word used in the selection-retrieval test.

This methodology was approved by the local University of Lincoln ethical review committee.

\section{Results}

\subsection{Comparative analysis of the model-rival technique, minimal stimulus enhancement, indirect stimulus enhancement, and direct stimulus enhancement}

Data on training and test times were checked for normality and their distributions were found to be unsuitable for parametric analysis. 
The training time was defined as the time taken to train the dog to correctly retrieve the target item from all three line-up positions during the selection-retrieval test. Each training session was $120 \mathrm{~s}$ in length, so each time a training session was repeated this time was added to the training time. Preliminary inspection of all data (including those training sessions that were failed) suggested that the direct stimulus enhancement method was the quickest training method to be learnt (median training time $(\mathrm{s})=540$ ), followed by the model-rival technique (median training time $(\mathrm{s})=840$ ), the minimal stimulus enhancement training method (median training time $(s)=960)$, and finally the indirect stimulus enhancement method which was the training method that took the longest to learn (median training time $(s)=1200)$.

The test time was defined as the time taken for the dog to retrieve the target item from all three line-up positions during the selection-retrieval test. This included the time taken in all incorrect selection-retrieval tests prior to the one in which the dog was successful. Timing began once the fetch command had been given and the dog was released, and ended when the dog had retrieved a toy. Data inspection suggested that the indirect stimulus enhancement training method had the quickest completion of the selection-retrieval test (median test time $(\mathrm{s})=92.5)$, followed by the direct stimulus enhancement training method (median test time $(s)=96$ ), and lastly the modelrival and minimal enhancement training methods (median test time $(\mathrm{s})$ for both methods $=119$ ).

The initial data were however censored due to the maximum time allowed for a response, therefore results from training sessions tests that were not passed were removed as these unsuccessful results might skew the results of further data analysis. Thus, for comparative purposes only the data from successfully passed training methods were used and these data are summarised in Table 1.

A cumulative binomial probability distribution was calculated to determine the significance of the number of dogs that successfully passed each training method. The probability of a dog passing the selection-retrieval test was calculated given the probability of a dog picking the target object in a line-up of three objects on three consecutive occasions and was $0.037\left((1 / 3)^{3}\right)$. As each dog had up to 10 attempts at the line-up, the probability that the dog would be successful within this time was 0.37 . As 10 dogs took part in the study, the cumulative binomial probability distribution of this probability suggests that if 7 or more dogs passed the selection-retrieval test the result would be considered significant with $P=0.036$.

Thus, only the model-rival and direct stimulus enhancement training methods had a significant number of dogs pass the selection-retrieval test, $(P=0.036$ for $7 \mathrm{dogs}$ in both training

Table 1

Summary statistics for the data collected for each training method, including only data from dogs that passed the training methods by successfully completing the selection-retrieval test for that training method (S.E.M.: standard error of mean)

\begin{tabular}{lllll}
\hline & Model-rival & $\begin{array}{l}\text { Minimal } \\
\text { enhancement }\end{array}$ & $\begin{array}{l}\text { Indirect stimulus } \\
\text { enhancement }\end{array}$ & $\begin{array}{c}\text { Direct stimulus } \\
\text { enhancement }\end{array}$ \\
\hline $\begin{array}{l}\text { Number of dogs that passed } \\
\text { Training time (s) }\end{array}$ & 7 & 6 & 2 & 7 \\
$\quad$ Mean \pm S.E.M. & $600 \pm 146$ & $520 \pm 157$ & $420 \pm 60$ & $480 \pm 114$ \\
Median & 480 & 420 & 420 & 360 \\
Range & $120-1200$ & $120-1080$ & $360-480$ & $120-1080$ \\
Test time (s) & & & & $115.1 \pm 31$ \\
Mean \pm S.E.M. & $161.1 \pm 46.9$ & $128 \pm 34.2$ & $92.5 \pm 5.5$ & 96 \\
Median & 119 & 119 & 92.5 & $17-259$ \\
Range & $24-344$ & $32-265$ & $87-98$ &
\end{tabular}


methods). The results from the minimal enhancement and indirect stimulus enhancement training methods were inconclusive, as the number of dogs successfully completing the retrievalselection test was 6 dogs for the minimal enhancement training method $(P=0.12)$ and 2 dogs for the indirect stimulus enhancement training method $(P=0.93)$.

A Kruskal-Wallis test on the successful training and test data from all four training methods revealed no significant difference between the four groups of training times $(H=0.47$, d.f. $=3$, $P=0.926)$ or the four groups of test times $(H=4.98$, d.f. $=3, P=0.173)$.

In order to investigate the rate of learning further in those training methods where 6 or more dogs successfully passed the selection-retrieval test, a second Kruskal-Wallis test was carried on the pass data for these trials. The data from the indirect stimulus enhancement method was removed for this second test due to the small number of dogs that successfully completed it which would have invalidated the analysis. The results of this analysis showed no significant differences between the training times for these three training methods $(H=0.36$, d.f. $=2, P=0.835)$, or the test times $(H=0.62$, d.f. $=2, P=0.735)$.

As both the model-rival and direct stimulus enhancement methods had a significant number of dogs that successfully passed the selection-retrieval test, the training and test times for these two training methods were compared using a Mann-Whitney $U$-test. This revealed no significant difference between the training times for these two training methods $(U=57.5, P=0.56)$. However, there was a significant difference between the test times for these two training methods ( $U=74, P=0.0072$ ), with the direct stimulus enhancement method having significantly shorter test times than the model-rival method.

Because individual dogs might have their own training and response style, a within subjects comparison was desirable. This was possible as 6 of the dogs in the study successfully passed both the model-rival and direct stimulus enhancement training methods. Thus, a Wilcoxon signed rank test was carried out on the data from these subjects to compare the training and test times for the model-rival training method with their corresponding training and test time for the direct stimulus enhancement training method. This revealed a significant difference between dogs training times for the model-rival and direct stimulus enhancement training methods $(T=36$, $P=0.014$ ), with the direct stimulus enhancement method having significantly quicker training times than the model-rival method. There was also a significant difference between the test times for these two methods $(T=21, P=0.036)$, with the direct stimulus enhancement method having significantly shorter test times than the model-rival method.

\subsection{Comparative analysis of data collected in this study and data collected in the McKinley and Young (2003) study}

The training times from the model-rival training method used in our study and from the model-rival training method in the McKinley and Young study were compared using a MannWhitney $U$-test. It was found that there was no significant difference between the training times of both studies as $U=61, P=0.92$.

The training times for our model-rival training method and McKinley and Young's operant training method were also compared using a Mann-Whitney $U$-test. This showed no significant difference between the time taken to train by these two methods as $U=62, P=0.83$.

The cumulative binomial probability distribution was also calculated for the number of dogs that apparently passed the model-rival and operant training methods in the McKinley and Young (2003) study, as this did not appear in their report. The probability that a dog correctly completed the selection-retrieval test was 0.37 , as calculated in our study. It was assumed that each dog had a 
maximum of 10 attempts at the line-up, as in our study, because after reviewing the data collected by McKinley and Young the maximum training time needed by any dog was within this time period. From McKinley and Young's report it appears that 15 dogs initially took part in their study, although the data from only 9 dogs that successfully passed both the model-rival and operant training methods was used in the final report. Assuming all $15 \mathrm{dogs}$ passed the operant training method (as this is a standard and reliable procedure), then only 9 could have passed the model-rival method (to only have 9 dogs passing both methods). The binomial probability of 9 out of $15 \mathrm{dogs}$ passing with this method is $P=0.059$. This result is not significant and questions the validity of their conclusion that the dogs learned with this method.

Finally, the training times for our direct stimulus enhancement training method were compared with the training times for McKinley and Young's operant training method using a Mann-Whitney $U$-test. It was found that there was no significant difference between the training times for these two methods as $U=54, P=0.596$.

\subsection{The discrimination test}

Out of the 8 dogs that took part in the discrimination test, 3 correctly retrieved the target object. To establish whether this was significant a cumulative binomial probability distribution was calculated. This was determined given that the probability of a dog randomly selecting the target object from a line-up of three objects was 0.33 and 8 dogs took part in the test. It was established that only if 6 or more dogs passed the discrimination test would the result be significant $(P=0.02)$. Thus, the results of the discrimination test were not significant $(P=0.53)$.

\section{Discussion}

The results of the study demonstrate that dogs are equally able to learn by some form of simple stimulus enhancement as by an adapted model-rival training method. As the model-rival and direct stimulus enhancement training methods had the same significant level of success in the selection-retrieval test, it is possible that the dogs were learning during the model-rival training sessions using simpler rules than the complex cognitive abilities implied by McKinley and Young's (2003) explanation of the success of this technique. Specifically the dogs might be learning from the interactions between the experimenters and the target object, rather than understanding the link between the experimenters' conversation and the target object.

The minimal enhancement and indirect enhancement training methods did not achieve a significant number of successful passes. However, the minimal enhancement method was close to reaching significance and so deserves further investigation with a larger sample size. Likewise, although the indirect stimulus enhancement method had a low pass rate in this study, the test and training times achieved by the dogs that did pass this training method were similar to those achieved in the other training methods. For this reason it is reasonable to suggest that this training method may also warrant further investigation with a larger sample size. Given the nearly significant low number of successful responders using this method it is possible that the dogs may have perceived the type of interaction between the experimenter and the target object in the indirect stimulus enhancement method as indicative of the object being aversive. It has been shown that dogs pay attention to, and track, the eye gaze of humans (Hare and Tomasello, 1999), so it is possible that as the experimenters were looking at the target object but not touching it themselves, this might have communicated an aversion to the object to the dog, and resulted in subsequent unwillingness to retrieve the object when later given a choice of objects. 
The training times from the direct stimulus enhancement training method used in this study were not found to be significantly different from those given by the model-rival training method when comparing the total data from the two training methods, however, from comparison of the training times of individual dogs succeeding with both methods it was found that the direct stimulus enhancement method was learnt significantly faster. The test times for the direct stimulus enhancement method were also found to be significantly quicker than those of the model-rival training method, both when comparing the total data from these methods and when comparing the performance of individual dogs that succeeded in both methods. This could suggest that the conversation used in the model-rival technique actually interfered with the dogs' learning as this verbal interchange is the main difference between the two training methods. Although it is possible that dogs are able to learn by the complex rules of the model-rival training method, it seems more likely that providing simpler cues during training is easier for the dog and achieves a faster rate of learning. Avoiding the use of the object name in any context other than retrieval may actually make learning easier for the dog as there is greater contiguity between the object name and the reward. By using the object's name frequently during training, without coupling it with a reward, contiguity is reduced possibly leading to interference in the learning of that object's name.

These results also suggest that dogs can learn object name associations even when the reward is delayed, i.e. when there is a low level of temporal contingency. With the exception of the model-rival method the first time that the dogs heard the name of the object was during the initial retrieval task following the stimulus enhancement procedure. Thus with the direct stimulus enhancement method, the successful dogs transferred their learning from the single correct response in the initial retrieval task of the training session to the three occasions in the selectionretrieval test afterwards.

Binomial probabilities for the number of dogs that passed McKinley and Young's model-rival method suggested that the number of dogs that successfully completed the selection-retrieval test might not have been significant. However, it should be noted the cumulative binomial probability distribution was based on the assumption that only 9 dogs passed the model-rival training method, given their results. Our more robust assessment of the data in the present study provides greater confidence in the conclusion that the dogs can learn the task with the model-rival method and their behaviour was not coincidental. This is reinforced by the comparison of the training times of our model-rival training method and McKinley and Young's model-rival training method which revealed no significant difference. It also meant that we could make comparisons between their results and ours using the model-rival training method as a point of reference. With this in mind a comparison was made between our model-rival training method and McKinley and Young's operant training method. This also revealed no significant differences in the training times of these two methods. This was not surprising as McKinley and Young (2003) found no difference between the training times of their model-rival and operant training methods, and their model-rival training times were similar to ours.

A final comparison between our direct stimulus enhancement training method and McKinley and Young's operant training method found that there was no significant difference between our direct stimulus enhancement training method and the traditional operant training method used by McKinley and Young (2003). However, our results suggest that within subjects comparisons may be necessary to avoid the risk of unsound conclusions. Basing conclusions on a lack of significance with a small sample size potentially leads to a type II statistical error especially when there appear to be individual differences in learning as revealed in the current study. 
The discrimination test revealed that dogs seem to generalise, or at least do not discriminate, their learning of object labels on the basis of colour. However, the phenomenon of discrimination is worthy of further investigation with a larger sample size, and investigating the discriminatory resolution of other object attributes such as size and related form.

In conclusion, it is suggested that the performance of dogs previously reported using a modified model-rival training method may reflect the use of simpler rules than those that underlie learning by the model-rival technique as described by Pepperberg (1999). It cannot be concluded that dogs necessarily understand the social complexities of such a situation at any level, as their attention may simply be drawn to the target object by observing the experimenters interacting with it. Indeed the conversation used may actually hinder the learning process. It is also concluded that once a dog has learnt the name of a particular object, it does not necessarily discriminate this object from other objects of the same shape but different colour.

\section{Acknowledgements}

Many thanks to UFAW for providing the vacation scholarship to Nina Cracknell that allowed this study to take place. We would also like to thank all of the owners who gave permission for their dogs to take part in the study.

\section{References}

Fukuzawa, M., Mills, D.S., Cooper, J.J., 2005. The effect of human command phonetic characteristics on auditory cognition in dogs (Canis familiaris). J. Comp. Psychol. 119, 117-120.

Galef, B.G., 1992. The question of animal culture. Hum. Nat. 3 (2), 157-178.

Hare, B., Tomasello, M., 1999. Domestic dogs (Canis familiaris) use human and conspecific social cues to locate hidden food. J. Comp. Psychol. 113, 173-177.

Kawai, M., 1965. Newly-acquired pre-cultural behavior of the natural troop of Japanese monkeys on Koshima islet. Primates 6 (1), 1-30.

McKinley, S., Young, R.J., 2003. The efficacy of the model-rival method when compared with operant conditioning for training domestic dogs to perform a retrieval-selection task. Appl. Anim. Behav. Sci. 81, 357-365.

Neitz, J., Geist, T., Jacobs, G.H., 1989. Colour vision in the dog. Vis. Neurosci. 3, 119-125.

Pearce, J.M., 1999. Animal Learning and Cognition. Psychology Press Ltd., East Sussex.

Pepperberg, I.M., 1999. The Alex Studies: Cognitive and Communicative Abilities of Grey Parrots. Harvard University Press, Cambridge, MA.

Schacter, D.L., 2001. The Seven Sins of Memory: How the Mind Forgets and Remembers. Houghton Mifflin, Boston. Wynne, C.D., 2001. Animal Cognition: The Mental Lives of Animals. Palgrave, New York. 